\title{
Verification of the homogeneity of the matrix/analyte mixture on sample plate using MALDI-MS technique and new ionic liquid matrices
}

\author{
Paulina Kobylis, Hanna Lis, Piotr Stepnowski, Magda Caban \\ Department of Environmental Analysis, Faculty of Chemistry, University of Gdańsk, Wita Stwosza 63, Gdańsk, Poland \\ Corresponding author: paulina.kobylis@phdstud.ug.edu.pl
}

DOI: $10.31708 /$ spi3.18/pkoby.cns18

\section{Introduction}

Matrix-assisted laser desorption/ionization (MALDI) is a soft ionization technique for mass spectrometry and usually is used in combination with the time of flight analyzer. This technique so far was used to investigation of large biomolecules. Due to the solid state of acid matrices and resulting problems with homogeneity of sample spot and low repeatability of analysis, and high background in low $\mathrm{m} / \mathrm{z}$ range the analysis of low molecular compound by MALDI-MS previously have been problematic. The breakthrough in this field occurred in 2001 after publishing a report by Armstrong at al. concerning ionic liquid matrices (ILMs) (Armstrong, Zhang, He, \& Gross, 2001). ILMs are organic salts, belongs to the second generation of ionic liquids formed by the reaction of an equimolar amount of a crystalline standard MALDI matrix with an organic base. This combination allows the transfer of proton from the acid to the base giving the resulting product nature of protic ionic liquid (Greaves \& Drummond, 2008). The aim of the research was verification of homogeneity of the matrix/analytical mixture on sample spot and the repeatability of the MALDI-TOF measurements. two selected standard (CHCA, HABA) and ionic liquid matrices (CHCA/TEA, HABA/a-MBA) were used. The experiment consisted of tested two methods of sample (matrix/analyte mixture) application on stain steel MALDI plate, namely dried droplet and thin layer. The examined group of low molecular weight compound were $\beta$-blockers $(\beta B), \beta$-agonist $(\beta A)$ and tricyclic antidepressants (TCA).

\section{Materials and Methods}

\subsection{The Chemicals}

The MALDI matrices a-cyano-4-hydroxycinnamic acid (CHCA), 2-(4-hydroxyphenylazo) benzoic acid (HABA) and $\alpha$-methylbenzylamine ( $\alpha-M B A)$, triethylamine (TEA) were purchased from Sigma-Aldrich (purity min. $98 \%$ ). The standards of $\beta$-blockers and $\beta$-agonists, tricyclic antidepressants (purity min. $98 \%$ ) were obtained from Sigma-Aldrich. The used organic solvent was as follow: methanol and acetonitrile (HPLC grade, $\mathrm{POCH}$, Poland).

\subsection{The matrix synthesis protocol}

The synthesis of ILMs was performed by simply protocol. The $20 \mathrm{mg}$ of acid was added to the $1.5 \mathrm{~mL}$ chromatographic vial and dissolved in $1 \mathrm{~mL}$ of mixture of $\mathrm{MeOH}$ : $\mathrm{ACN}(1: 1, \mathrm{v} / \mathrm{v})$. Then the equimolar volume of amine was added. After vortex mixing ( $5 \mathrm{~min}$ ), and 1 hour standing in room temperature, the solvent was evaporated by the nitrogen stream in $60^{\circ} \mathrm{C}$.

\subsection{MALDI analysis and matrix preparation}

All matrices after synthesis were dissolved in $1 \mathrm{~mL}$ of mixture of ACN: H2O (2:1, v/v). Then the MALDI-MS measurements was consisted of application around $1 \mu \mathrm{L}$ mixture of the matrix and the selected analyte $(\beta B, \beta A$ and TCA) in concentration $50 \mu \mathrm{g} / \mathrm{ml}$ on a MALDI plate using dried droplet and thin layer method. After evaporation of mixture of solvents, the plate has been analyzed. Each circular sample spot was divided approximately into nine area parts with a grid. The analysis was performed using MALDI TOF/TOF 5800+ mass spectrometer (AB SCIEX), equipped with $\mathrm{Nd}$ : YAG, $266 \mathrm{~nm}$ pulsed laser $(1 \mathrm{kHz})$. During the measurement, the number of laser shots usually were 400 . The mass spectra were recorded in positive-ion reflection mode in specifically determinate place on plate.

\section{Results and Discussion}

Based on the obtained results, it was found that the choice of the proper matrix (standard or ionic liquid matrix) and the method of application to the plate (the dried droplet and thin layer method) affects the result of MALDI-MS analysis (homogeneity and repeatability). Moreover, it was noted that the best precision values in the range of 13 to $17 \%$ were obtained for $\beta B$ and $\beta A$ using $\mathrm{CHCA} / \mathrm{TEA}$ and thin layer method. Similar results for the same group of analytes were obtained for analysis by the standard CHCA matrix. However, the precision values obtained for all analytes are not as similar as in the case of CHCA/TEA. Furthermore, it was also found that in the case of the second liquid matrix HABA/a-MBA the best precision values were obtained for TCA using the thin layer method in the range of $14-19 \%$. What's more the results obtained for $\beta B$ and $\beta A$ and TCA using the 
standard HABA matrix were not satisfactory due to the fact that the precision value significantly exceeded $15 \%$.

\section{Conclusions}

The obtained precision for analysis of small molecules by the ILMs was higher than expected and higher than those obtained in the literature reports. Still, some patterns were determinated. These studies contribute to the broadening of knowledge regarding the proper selection of the matrix for the potential analyte and allow a better understanding of the MALDI ionization mechanism when the ionic liquid matrices are applicable. What's more, they are a prelude to further research with special emphasis on interactions not only in the matrix itself, but also between the matrix and the analyte.

\section{Acknowledgments}

Financial support was provided by National Science Centre under grand grant UMO-2017/25/N/ST4/02390.

\section{References}

Armstrong, D. W., Zhang, L. K., He, L., \& Gross, M. L. (2001). Ionic liquids as matrixes for matrixassisted laser desorption/ionization mass spectrometry. Analytical Chemistry, 73(15), 36793686. https://doi.org/10.1021/ac010259f

Greaves, T. T. L., \& Drummond, C. J. C. (2008). Protic ionic liquids: Properties and applications. Chemical Reviews. American Chemical Society. https://doi.org/10.1021/cr068040u 